Kenji Shigemi, MD

Department of Anesthesiology \& Reanimatology

University of Fukui

Faculty of Medicine Sciences

Fukui, Japan

E-mail: kshigemi@u-fukui.ac.jp

\section{References}

1. Rosenstock J, Tuchman M, LaMoreaux L, Sharma U. Pregabalin for the treatment of painful diabetic peripheral neuropathy: A double-blind, placebo-controlled trial. Pain 2004; 110:628-638.

2. Smith HS, Harris R, Clauw D. Fibromyalgia: An afferent processing disorder leading to a complex pain generalized syndrome. Pain Physician 2011; 14:E217E245.

3. Freynhagen R, Strojek K, Griesing $T$, Whalen E, Balkenohl M. Efficacy of pregabalin in neuropathic pain evaluated in a 12-week, randomized, double-blind, multicentre, placebo-controlled trial of flexible-and fixed-dose regimens. Pain
2005; 115:254-263.

4. Gilron I. Gabapentin and pregabalin for chronic neuropathic and early postsurgical pain: Current evidence and future directions. Curr Opin Anaesthesiol 2007; 8. 20:456-472.

5. Bockbrader HN, Wesche D, Miller R, Chapel S, Janiczek N, Burger P. A comparison of the pharmacokinetics and 9 . pharmacodynamics of pregabalin and gabapentin. Clin Pharmacokinet 2010; 49:661-669.

6. Hellwig S, Amtage F. Pregabalin- induced cortical negative myoclonus in a patient with neuropathic pain. Epilepsy Behav 2008; 13:418-420.
7. Perez Lloret S, Amaya M, Merello $M$. Pregabalin-induced parkinsonism: A case report. Clin Neuropharmacol 2009; 32:353-354.

8. Martí Massó JF, Obeso JA, Carrera N, Martínez-Lage JM. Aggravation of Parkinson's disease by cinnarizine. J Neurol Neurosurg Psychiatry 1987; 50:804-805.

9. Pileblad E, Carlsson A. In vivo effects of the Ca2+-antagonist nimodipine on dopamine metabolism in mouse brain. J Neural Transm 1986; 66:171-187.

10. Terland O, Flatmark T. Drug-induced parkinsonism: Cinnarizine and flunarizine are potent uncouplers of the vacuolar $\mathrm{H}+-$ ATPase in catecholamine storage vesicles. Neuropharmacology 1999; 3.

\title{
Caudal Epidural Injections for the Treatment of Tarlov Cysts: Suggestions for the Better Results
}

\section{To THE EDITOR}

It is with great interest that we read the case report by Freidenstein et al, "Minimally invasive interventional therapy for Tarlov cysts causing symptoms of interstitial cystitis," published in the 2012 March/April issue of Pain Physician (1).

This is a well organized and prepared case report which showed successful result in treating Tarlov cysts with symptoms similar to interstitial cystitis. To treat these cysts in this article, authors performed caudal epidural injections by inserting the needle only a few millimeters through the sacral hiatus and administering $3 \mathrm{ml}$ of $1 \%$ lidocaine and $40 \mathrm{mg}$ of triamcinolone. Authors thought that the reduction of inflammation around the cysts may lessen the cyst distention and subsequent pressure on the nearby nerve root. In ad- dition, they recommended to avoid dural puncture during the procedure and to use low-volume drugs to prevent inadvertent increase in pressures. We applaud the authors' work, however, we have some different points of view on the injection method and the volume of injectate.

First, we believe that the injection method should be adjusted according to the location and size of Tarlov cysts. Because the cyst itself or the secondary inflammation hinders the delivery of steroid and local anesthetic agent to the target lesion, it would be more effective to inject as near as possible to the cysts. In addition, Tarlov cysts possibly cause symptoms compressing or irritating nearby nerve roots as the herniated intervetebral discs (2). Also, caudal epidural injections need large volume 
of drug to spread to upper segments of spine (3), so about $3 \mathrm{ml}$ of drugs may be not enough to reach from the sacral hiatus to the target lesion. Therefore, in cases of Tarlov cysts located far from the sacral hiatus, transforaminal approach or caudal approach with catheterization will be more appropriate.

Second, a large-volume injectate seems more effective than the small one. As Mitra et al (4) commented in his case report, inflammatory component of Tarlov cysts contribute to the pathogenesis. Therefore, steroid injection with enough volume will be better at washing out inflammatory mediators and relieving the inflammation-induced epidural fibrosis (5). In our experience, we successfully and safely treated the Tarlov cysts causing perineal and peri-anal pain with a series of sacral transforaminal steroid injections administering $8 \mathrm{ml}$ of normal saline followed by $8 \mathrm{~mL}$ of $0.19 \%$ ropivacaine and $10 \mathrm{mg}$ of triamcinolone (Fig. 1).

In conclusion, we suggest a feasible approach to the target lesion and an enough volume injection anticipating epidural adhesiolysis and effective drug delivery.
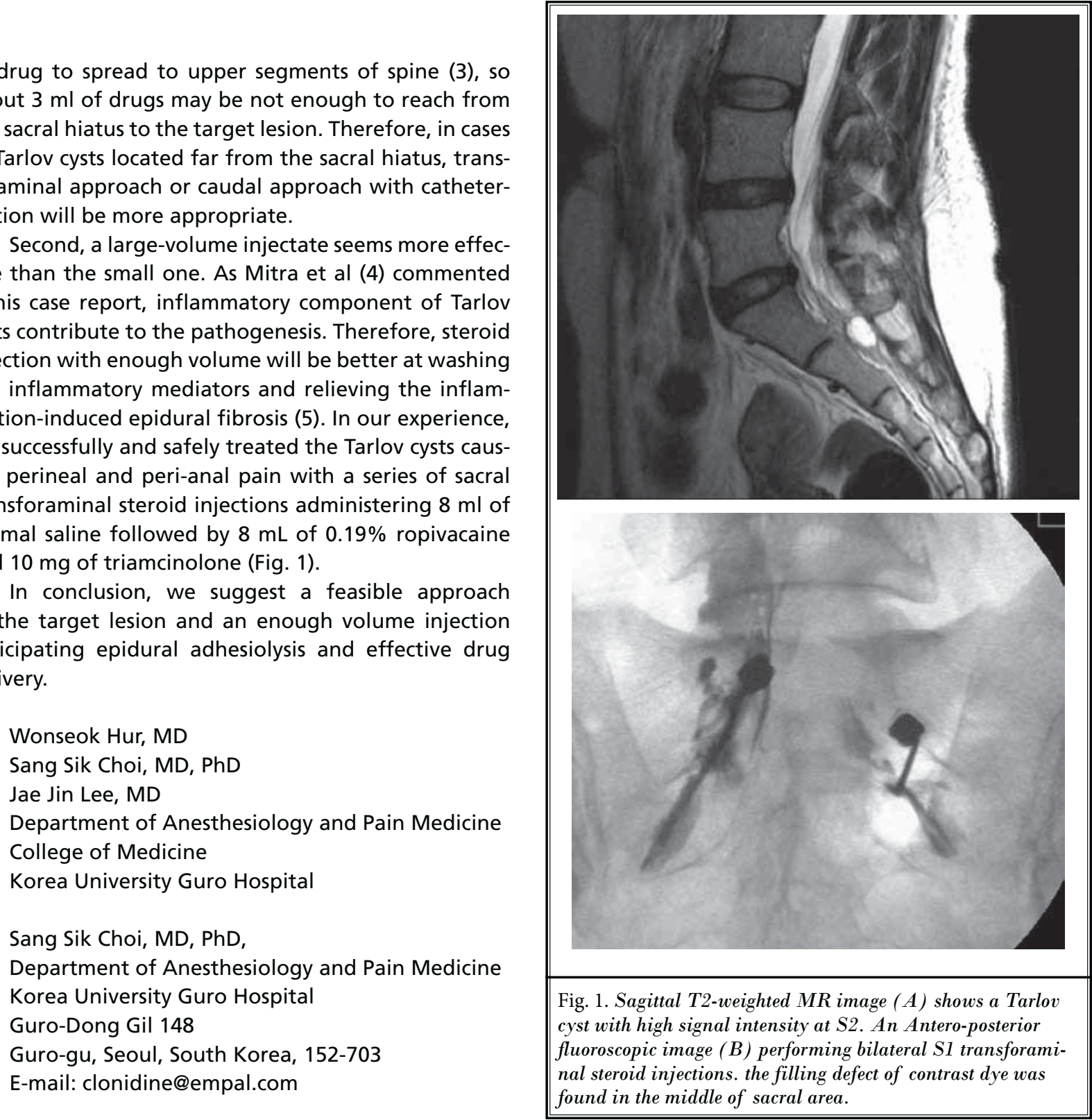

\section{References}

1. Freidenstein J, Aldrete JA, Ness T. Minimally invasive interventional therapy for Tarlov cysts causing symptoms of interstitial cystitis. Pain Physician 2012; 15:141146.

2. Tarlov IM. Spinal perineurial and meningeal cysts. J Neurol Neurosurg Psychiatry
1970; 33:833-843.

3. Singh V, Manchikanti L. Role of caudal epidural injections in the management of chronic low back pain. Pain Physician 2002; 5:133-148.

4. Mitra R, Kirpalani D, Wedemeyer M. Conservative management of perineural cysts. Spine (Phila Pa 1976) 2008; 33:E565568.

5. Racz GB, Heavner JE, Trescot A. Percutaneous lysis of epidural adhesions-evidence for safety and efficacy. Pain Practice 2008; 8:277-286. 


\section{Response}

While we agree that the route and volume of injectate depend on the precise location of the cyst, we take the stance that it is of paramount importance to avoid cyst puncture when performing interventional therapy on Tarlov Cysts. It is for this reason that we highlight advancing the needle only a few millimeters past the sacral hiatus. This is why our primary focus has been on addressing lower level sacral cysts. The risks of cyst puncture include persistent dural leak, which can be extremely difficult to seal as standard blood patches can be ineffective and possibly increase the risk of adhesive arachnoiditis. This risk is compounded by the possibility of injecting particulate steroids intrathecally when working directly around cysts. Having said that, in order to target higher level cysts, this may be a necessary risk. It is encouraging to hear that other practitioners are having success with interventional therapy for Tarlov Cysts. We believe that these have been dismissed as incidental findings for decades and can represent an occult cause of chronic pain.

James Freidenstein, MD

Ambulatory Care Pain Specialists

220 Fort Sanders West Blvd

Bldg 2, Suite 200

Knoxville, TN 37922

email: freidenstein@hotmail.com

\section{In Errata}

The coauthor of the letter to the editor titled Does the Diagnosis of Spondylolisthesis Matter published in the March/April issue of Pain Physician journal (2012;15:E157) was inadvertently left off.

The letter was also submitted by Nilay Chatterjee, MD, DM, a Consultant in the Department of Pain Medicine at the Institute of Neurosciences, Kolkata West Bengal, India 
